\title{
Das «mittlere Einkommen» im Sinne von Art. 65 Abs. 1 bis KVG
}

Besprechung des Urteils des Bundesgerichts 8C_228/2018 vom 22. Januar 2019 (zur Publikation vorgesehen)

Andreas Petrik *

Das Bundesrecht sieht für untere und mittlere Einkommen eine prozentuale Verbilligung der Krankenkassenprämien von Kindern und jungen Erwachsenen in Ausbildung vor. Liegt die durch einen Kanton festgelegte Einkommensgrenze nur in einem geringen Ausmass über der Schwelle zwischen den unteren und mittleren Einkommen, ist dies mit den bundesrechtlichen Vorgaben nicht vereinbar.

I. Sachverhalt 27

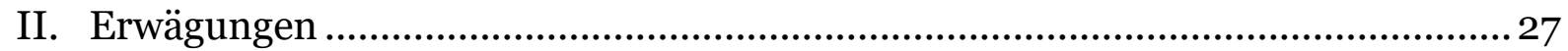

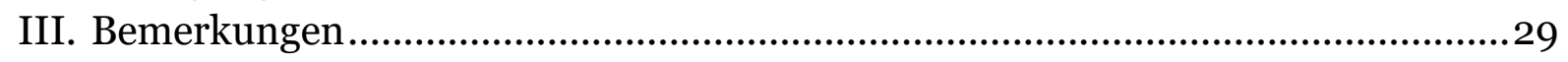

Zitiervorschlag: Andreas Petrik, Das «mittlere Einkommen» im Sinne von Art. 65 Abs. $1^{\text {bis }}$ KVG, in: sui-generis 2019, S. 26

URL: $\quad$ sui-generis.ch/90

DOI: $\quad$ https://doi.org/10.21257/sg.90

* Andreas Petrik ist als Rechtsanwalt vorwiegend in den Bereichen Arbeits- und Versicherungsrecht tätig. Als wissenschaftlicher Mitarbeiter im Zentrum für Sozialrecht (ZSR) der Zürcher Hochschule für angewandte Wissenschaften (ZHAW) unterrichtet er Arbeits- und Sozialversicherungsrecht, andreas.petrik(at)petrikpartner.ch.

Dieses Werk ist lizenziert unter einer Creative Commons Namensnennung - Weitergabe unter gleichen Bedingungen 4.o International Lizenz. 


\section{Sachverhalt}

1 Der Kanton Luzern gewährte auf die Krankenkassenprämien von Kindern bis zum vollendeten 18. Lebensjahr und von jungen Erwachsenen bis zum 25. Lebensjahr in Ausbildung im Jahre 2016 eine Verbilligung in der Höhe von 50 \%, soweit das massgebende Einkommen den Betrag von CHF 75'ooo.- nicht überstieg. Mangels eines definitiven Voranschlags für das Jahr 2017 beschloss der Regierungsrat einen lediglich provisorischen Anspruch auf Prämienverbilligung in der Höhe von 75 \% des errechneten Betrages. Die Einkommensgrenze von CHF 75'ooo.blieb unverändert. Nach Verabschiedung des definitiven Budgets im September 2017 setzte der Regierungsrat die Einkommensgrenze rückwirkend auf den 1. Januar 2017 auf CHF 54'O00.- fest. Ausserdem wurde eine Bestimmung aufgenommen, wonach allfällige Differenzen zwischen dem provisorischen und dem definitiven Anspruch von der Ausgleichskasse an die Krankenversicherer zurückerstattet werden mussten.

Mehrere Personen verlangten beim Kantonsgericht des Kantons Luzern unter anderem die Aufhebung der geänderten Verordnungsbestimmungen, die die Herabsetzung der Einkommensgrenzen zum Gegenstand hatte. Das Kantonsgericht erkannte, dass die vom Regierungsrat festgesetzte Einkommensgrenze nicht gegen die bundesrechtlichen Vorgaben verstosse. ${ }^{1}$

3 Die Beschwerdeführer erhoben gegen den Entscheid des Kantonsgerichtes Beschwerde beim Bundesgericht und ver-

Entscheid vom 20. Februar 2018, LGVE 2018 III Nr. 1. langten unter anderem die Aufhebung der Bestimmungen betreffend die Herabsetzung der Einkommensgrenze. Das Bundesgericht heisst die Beschwerde gut.

\section{Erwägungen}

4 Gemäss den Ausführungen des Bundesgerichtes habe der Bundesgesetzgeber die Prämienverbilligung föderalistisch ausgestaltet. Damit sei es Sache der Kantone, den Kreis der Begünstigten, die Höhe, das Verfahren und den Auszahlungsmodus festzulegen. Es stehe den Kantonen frei zu entscheiden, wie der für die Prämienverbilligung zur Verfügung stehende Gesamtbetrag verteilt werden soll; es sei möglich, einer geringeren Anzahl von Berechtigten einen höheren oder einer grösseren Anzahl einen tieferen Beitrag zu leisten. Schliesslich könnten die Kantone definieren, was unter «bescheidenen wirtschaftlichen Verhältnissen» und «unteren und mittleren Einkommen» zu verstehen sei (E. 3.2). Die Autonomie der Kantone sei jedoch insofern beschränkt, als die Ausführungsbestimmungen nicht gegen Sinn und Geist der Bundesgesetzgebung verstossen und deren Zweck nicht beeinträchtigen dürften (E. 3.2).

5 Das Bundesgericht hält fest, dass das Bundesrecht den Kantonen gemäss Art. 65 Abs. $1^{\text {bis }}$ KVG vorschreibe, die Prämien von Kindern und jungen Erwachsenen für untere und mittlere Einkommen um mindestens $50 \%$ zu verbilligen. ${ }^{2}$ Keine bundesrechtlichen Vorgaben bestünden jedoch hinsichtlich des Ein-

2 Gemäss der aktuellen Fassung von Art. 65 Abs. $1^{\text {bis }}$ KVG haben die Kantonen die Prämien für Kinder um $80 \%$ und jene von jungen Erwachsenen in Ausbildung um $50 \% \mathrm{zu}$ verbilligen. 
kommensbegriffs und der Frage, was betragsmässig unter «unteren und mittleren Einkommen» zu verstehen sei. Im Unterschied zum Begriff der «bescheidenen wirtschaftlichen Verhältnissen» im Sinne von Art. 65 Abs. 1 KVG, der die bundesrechtlichen Vorgaben für die Prämienverbilligung von erwachsenen Personen umschreibe, sähe Abs. $1^{\text {bis }}$ auch eine Verbilligung der Prämien für «mittlere Einkommen» vor. Relevant für die streitige Frage der Bundesrechtskonformität der Einkommensgrenze von $\mathrm{CHF}$ 54'ooo.- sei deshalb die Auslegung des Begriffs der «mittleren Einkommen» (E. 6.1).

In einem ersten Schritt geht das Bundesgericht auf die Entstehungsgeschichte von Art. 65 Abs. $1^{\text {bis }}$ KVG ein. Das Parlament habe den Vorschlag des Bundesrates abgelehnt, der eine nach Einkommen gestaffelte Prämienverbilligung durch Festlegung des Eigenanteils an den Krankenkassenprämien in Prozenten des Gesamteinkommens vorgesehen habe (E. 6.2.2). Unter Bezugnahme auf verschiedene Voten im National- und Ständerat kommt das Bundesgericht zum Schluss, dass sich die eidgenössischen Räte entschieden hätten, auf eine Definition des Begriffs «untere und mittlere Einkommen» zu verzichten. Aus den Äusserungen in den Räten werde jedoch auch ersichtlich, dass der Gesetzgeber nicht nur die unteren, sondern eben auch die mittleren Einkommen begünstigen wollte (E. 6.2.3).

7 Das Bundesgericht zeigt im Folgenden die Entwicklung der Ausgestaltung der Prämienverbilligung im Kanton Luzern auf. Die Umsetzung der bundesrechtlichen Vorgaben, wonach die Prämien für
Kinder und junge Erwachsenen in Ausbildung um $50 \%$ zu verbilligen sind, sei im Kanton Luzern zunächst ohne Einkommensgrenze erfolgt; die im Bundesrecht vorgesehenen Verbilligung wurde unabhängig vom Einkommen gewährt (E. 7.2.1). Ab dem 1. Januar 2008 habe gemäss der einschlägigen Verordnung eine Einkommensgrenze von $\mathrm{CHF}$ 100'Ooo.- gegolten (E. 7.2.2). Auf den 1. Juli 2013 habe der Regierungsrat die Verordnung dahingehend geändert, dass bis zu einer Einkommensgrenze von CHF 80'ooo.- die Prämien von Kindern und jungen Erwachsenen in Ausbildung verbilligt wurden, sofern die Prämien das anrechenbare Einkommen um mindestens $10 \%$ überstiegen (E. 7.2.3). Per 1. Januar 2015 sei die Einkommensgrenze auf CHF 75'0oo.- und per 1. Januar 2017 auf CHF 54'Ooo.- gesenkt worden (E. 7.2.4 f.).

8 Bezugnehmend auf die Begründung des kantonalen Gerichtes führt das Bundesgericht aus, dass dieses im Rahmen einer Plausibilitätsprüfung die Einkommen von Verheirateten mit Kindern, die zwischen $70 \%$ und $150 \%$ des Medianwertes lägen, als «mittlere Einkommen» angesehen habe. Ausgehend von einem Medianwert von CHF 86'875.- ergäbe sich eine Bandbreite von CHF 6o'812.50 bis CHF 130'312.50. Unter Berücksichtigung des in der kantonalen Verordnung zur Prämienverbilligung vorgesehenen Abzuges für Kinder und junge Erwachsenen in Ausbildung in der Höhe von CHF 9'ooo.liege die Einkommensgrenze damit bei CHF 63'000.- (CHF 54'00o.- + $\mathrm{CHF}$ 9'ooo.-) - und damit über dem Wert, der $70 \%$ des Medianwertes entspreche. Für Verheiratete mit zwei Kindern bestehe unter Berücksichtigung des Abzuges bis 
zu einem Einkommen von CHF 72'Ooo.Anspruch auf Prämienverbilligung. Aufgrund dieser Erwägungen sei die Vorinstanz zum Schluss gekommen, dass nicht nur Familien mit tiefen Einkommen, sondern auch Familien mit mittleren Einkommen in den Genuss der Prämienverbilligung für Kinder und junge Erwachsenen in Ausbildung kommen würden (E. 8.2).

9 Nach Ansicht des Bundesgerichtes vermögen die Ausführungen des kantonalen Gerichtes nicht zu überzeugen. Als Haushalte der unteren Mitte würden jene gelten, die ein Einkommen zwischen $70 \%$ (CHF 6o'812.50) und $100 \%$ (CHF 86'875.-) des Medianwertes erzielen würden. Ein Einkommen von $\mathrm{CHF}$ 63'ooo.- liege ganz knapp über der Schwelle von den unteren Einkommen zu denjenigen der unteren Mitte. Damit habe nur ein ausgesprochen geringer Teil der Haushalte mit einem mittleren Einkommen Anspruch auf Prämienverbilligung für Kinder und junge Erwachsenen in Ausbildung. Dies widerspreche Sinn und Geist von Art. 65 Abs. $1^{\text {bis }}$ KVG, da dieser gerade auch für mittlere Einkommen eine Entlastung von den Krankenkassenprämien bringen sollte. Finanzpolitisch motivierte Herabsetzungen der Einkommensgrenze seien nicht per se ausgeschlossen, die zuletzt vorgenommene Herabsetzung verstosse jedoch gegen Bundesrecht (E. 8.3.3).

Die Beeinträchtigung des Zweckes der Bundesgesetzgebung ergebe sich des Weiteren aus der Tatsache, dass der Kantonsanteil mit der Herabsetzung der Einkommensgrenze von $35,9 \%$ im Jahr 2010 auf 19,2 \% im Jahr 2017 gesunken sei. Dies widerspreche der Vereinbarung zur Neuausgestaltung des Finanzausgleichs und der Aufgabenteilung zwischen Bund und Kantonen (NFA), die einen etwa hälftigen Beitrag von Bund und Kantonen an die Gesamtkosten für die Prämienverbilligung vorsehen würde. Der vom Kanton getragene Anteil beziehe sich zwar auf die Prämienverbilligung im Allgemeinen und nicht nur auf die vorliegend relevanten Familien mit Kindern und jungen Erwachsenen in Ausbildung. Dennoch würde sich auch vor diesem Hintergrund auf einen Verstoss gegen Sinn und Geist der Bundesgesetzgebung schliessen lassen (E. 8.3.4).

11 Im Ergebnis hebt das Bundesgericht die kantonalen Verordnungsbestimmungen auf, soweit diese eine Herabsetzung der Einkommensgrenze auf CHF 54'ooo.- für Familien mit Kindern und jungen Erwachsenen in Ausbildung vorsehen. Das Bundesgericht hält ausserdem fest, dass auch die in der kantonalen Verordnung vorgesehene Pflicht zur Rückzahlung von gestützt auf die altrechtliche Bestimmung provisorisch im Jahr 2017 ausgerichteten Prämienverbilligungen als aufgehoben gelte. Da diese Pflicht in direktem $\mathrm{Zu}-$ sammenhang mit den aufgehobenen Bestimmungen zur Einkommensgrenze stehe, würde diese in der Folge jeglichen Sinnes entleert.

\section{Bemerkungen}

12 Das Bundesgericht hatte sich mit der Frage auseinanderzusetzen, was unter dem Begriff «mittlere Einkommen» im Sinne von Art. 65 Abs. $1^{\text {bis }}$ KVG zu verstehen ist. Eine betragsmässige Festlegung findet sich dabei weder im kantonalen Recht noch im Bundesrecht. Wie auch das kantonale Gericht stützt sich 
das Bundesgericht auf eine Definition, die das Bundesamt für Statistik einer der Studie «Die Mitte im Fokus - Die Entwicklung der mittleren Einkommensgruppen von 1998 bis 2009»3 zu Grunde gelegt hat. Ausgangspunkt der Definition des Begriffs «mittlere Einkommen» bildet der sogenannte Medianwert. Im $\mathrm{Zu}$ sammenhang mit der Höhe des Einkommens liegen die Hälfte aller erfassten Einkommen über und die andere Hälfte unter dem Medianwert. Als «mittlere Einkommen» wurden jene definiert, die zwischen $70 \%$ und $150 \%$ des Medianwertes bewegen.

Gemäss Auffassung des kantonalen Gerichtes erfolgte die Festlegung der Einkommensgrenze durch den Regierungsrat im Einklang mit den bundesrechtlichen Vorgaben. Dem widerspricht das Bundesgericht und verwendet dabei deutliche Worte. Die Ausführungen des kantonalen Gerichtes, wonach 70 \% des Medianwertes einem Einkommen von CHF 6o'812.50 entsprechen und gemäss der revidierten Verordnung - unter Berücksichtigung des Kinderabzuges bei der Berechnung des für die Prämienverbilligung relevanten Einkommen - Familien mit einem Einkommen bis CHF 63'000.- in den Genuss von Prämienverbilligungen für Kinder und junge Erwachsene in Ausbildung kommen würden - womit eben auch mittleren Einkommen unterstützt würden, werden vom Bundesgericht als widersprüchlich bezeichnet. Dem ist insofern beizupflichten, als es kaum nachvollziehbar scheint, wenn daraus der Schluss gezogen wird, der Kanton Luzern

Bundesamt für Statistik (Hrsg.), Die «Mitte» im Fokus - Die Entwicklung der mittleren Einkommensgruppen von 1998 bis 2009, Neuchatêl 2013. komme seiner Pflicht nach, die Prämien für Kinder und junge Erwachsenen in Ausbildung für untere und mittlere Einkommen im vorgeschriebenen Ausmass $\mathrm{zu}$ verbilligen, da das kantonale Gericht selber festhält, dass Einkommen bis CHF 130'312.50 als «mittlere Einkommen» gelten würden.

14 Die Frage, wo die Untergrenze der «mittleren Einkommen» im Sinne von Art. 65 Abs. $1^{\text {bis }}$ KVG liegt, wird vom Bundesgericht freilich nicht beantwortet. Der Bundesgesetzgeber überliess die Festlegung der Einkommensgrenzen den Kantonen. Darüber, wie tief die Kantone die Einkommensgrenze festlegen können, ohne den vom Bundesrecht gesetzten Rahmen zu sprengen, kann nur spekuliert werden. Der Regierungsrat des Kantons Luzern hat die Einkommensgrenze mit Beschlüssen vom 5. Februar $2019^{4}$ für die Jahre 2017 bis 2019 auf CHF 78'154.- festgelegt. Unter Berücksichtigung des im kantonalen Prämienverbilligungsgesetz vorgesehenen Abzuges für ein Kind in der Höhe von CHF 9'ooo.- entspricht dieser Betrag einem steuerbaren Einkommen von $\mathrm{CHF}$ 87'154.--

15 Dieser Betrag entspricht exakt dem aktuellen Medianwert der Reineinkommen von Verheirateten mit Kindern5. Der Regierungsrat des Kantons Luzern hat demnach die Einkommensgrenze für den Anspruch auf Prämienverbilligung dem Medianwert gleichgestellt. Den Anforderungen des Bundesrechts wird damit sicher genüge getan, wobei davon auszugehen ist,

$4 \overline{\text { Luzerner Kantonsblatt Nr. } 6 \text { vom 9. Februar }}$ 2019, S. 375 f.

5 Siehe auch die Verteilung des Reineinkommens nach Alter, Zivilstand und Kindern 2016 (Stand August 2018). 
dass auch eine unter dem Median liegende Einkommensgrenze mit den bundesrechtlichen Vorgaben vereinbar gewesen wäre. 